\title{
Perspective
}

\section{Diverse Systems for Efficient Sequence Insertion and Replacement in Precise Plant Genome Editing}

\author{
Yingxiao Zhang ${ }^{1}$ and Yiping Qi $\mathbb{1}^{1,2}$ \\ ${ }^{1}$ Department of Plant Science and Landscape Architecture, University of Maryland, College Park, Maryland 20742, USA \\ ${ }^{2}$ Institute for Bioscience and Biotechnology Research, University of Maryland, Rockville, Maryland 20850, USA \\ Correspondence should be addressed to Yiping Qi; yiping@umd.edu
}

Received 23 June 2020; Accepted 19 July 2020; Published 28 July 2020

Copyright ( 2020 Yingxiao Zhang and Yiping Qi. Exclusive Licensee Nanjing Agricultural University. Distributed under a Creative Commons Attribution License (CC BY 4.0).

\begin{abstract}
CRISPR-mediated genome editing has been widely applied in plants to make uncomplicated genomic modifications including gene knockout and base changes. However, the introduction of many genetic variants related to valuable agronomic traits requires complex and precise DNA changes. Different CRISPR systems have been developed to achieve efficient sequence insertion and replacement but with limited success. A recent study has significantly improved NHEJ- and HDR-mediated sequence insertion and replacement using chemically modified donor templates. Together with other newly developed precise editing systems, such as prime editing and CRISPR-associated transposases, these technologies will provide new avenues to further the plant genome editing field.
\end{abstract}

\section{Main Text}

Clustered Regularly Interspaced Short Palindromic Repeats(CRISPR-) mediated genome editing is a powerful and versatile tool for manipulating nucleic acids. However, targeted sequence insertion and replacement remains a significant challenge in plants. The efficiency of homology-directed repair (HDR) is usually low and inconsistent; as in plant somatic tissues, the predominant pathway to repair doublestrand breaks (DSBs) is nonhomologous end joining (NHEJ). Many attempts have been made to improve HDR efficiencies, including actively selecting desired sequences (such as selective markers) [1-3], manipulating repair pathways [4], enriching donor templates near Cas nucleases [5], and increasing the amount of donor templates by simply providing more template or by using novel strategies such as geminivirus replication [6-8] and RNA transcription [9]. NHEJmediated gene insertion has also been demonstrated in plants with low editing efficiencies [10]. To develop a robust and efficient method to achieve targeted sequence insertion and replacement in plants, Lu et al. has improved the NHEJmediated DNA insertion approach using chemically modified donor DNA (Figure 1(a)) [11]. Furthermore, this approach has been leveraged to develop a tandem repeatfacilitated HDR strategy (TR-HDR) (Figure 1(b)) [11]. This study significantly increased the efficiencies of NHEJ- and HDR-mediated DNA insertion and replacement when compared to previous studies. The demonstrated methodology should be applicable in other plants and enable precise genome editing in basic plant research and crop breeding.

$\mathrm{Lu}$ et al. first focused on NHEJ-mediated gene insertion. To improve the double-stranded DNA (dsDNA) donor stability, two phosphorothioate linkages were added at the $5^{\prime}$ and $3^{\prime}$ ends of both DNA strands. In addition, $5^{\prime}$-phosphorylation has been used to facilitate the NHEJ repair [11, 12]. Significantly higher insertion efficiencies were observed using modified dsDNA compared to unmodified dsDNA and single-stranded DNA (ssDNA). When targeting multiple genes separately or simultaneously using short $(<70 \mathrm{bp})$ modified dsDNA donors, high efficiencies (10.6\%-47.3\%) were observed, suggesting this approach is highly robust and efficient. Furthermore, longer donors with lengths of $526 \mathrm{bp}$ and 2,049 bp were simultaneously inserted into two loci, with $25.5 \%$ and $10.5 \%$ combined (two loci) insertion frequencies, respectively, indicating this approach is capable of inserting long DNA fragments. 


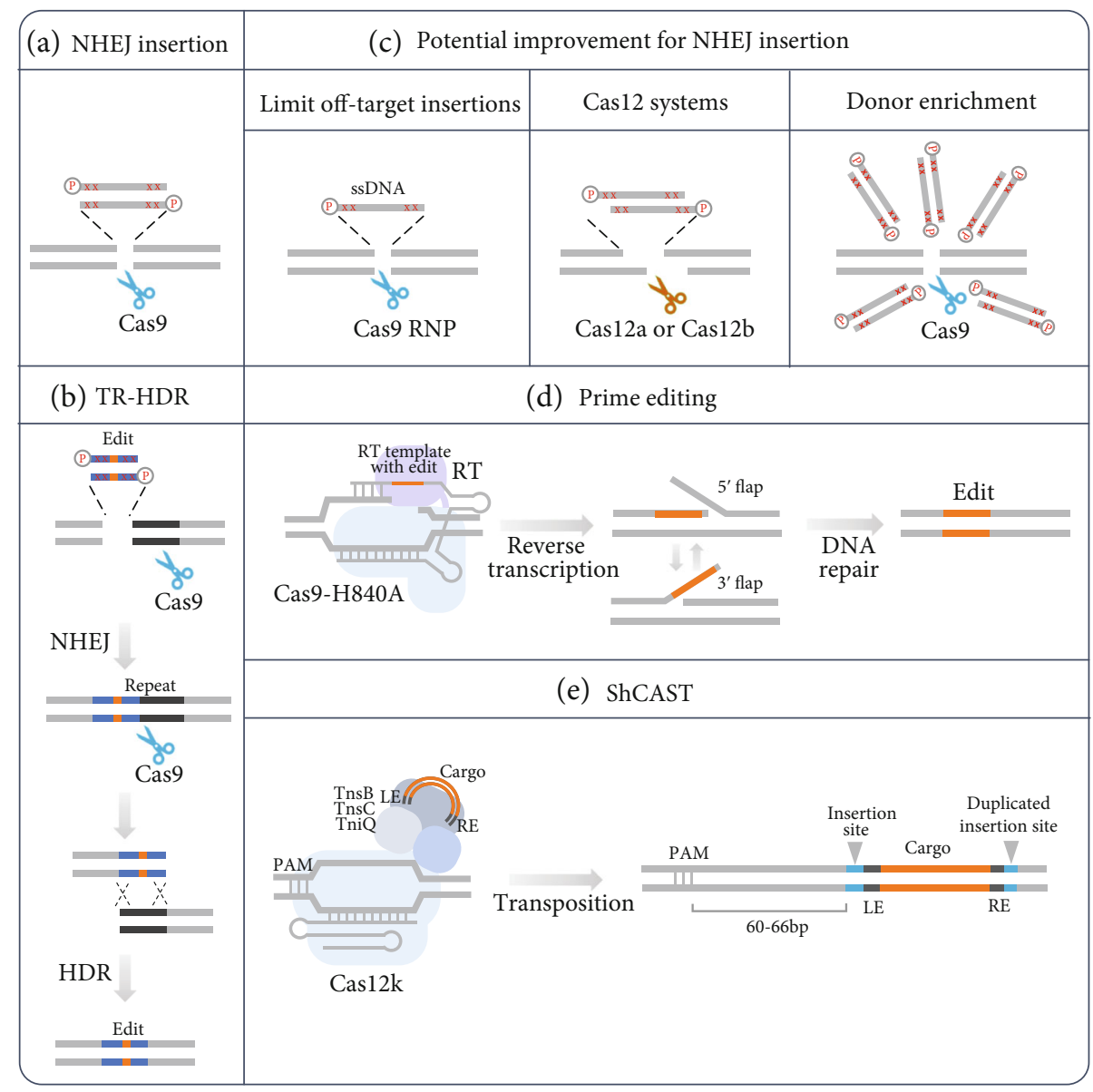

FIGURE 1: Schematic illustration of targeted sequence insertion and replacement technologies. (a) NHEJ-mediated targeted sequence insertion using chemically modified DNA donor templates. (b) Targeted sequence replacement using the tandem repeat-HDR strategy (TR-HDR). (c) Potential improvement for NHEJ-mediated sequence insertion, including using ssDNA or RNP to minimize off-target insertions, using the Cas12a or Cas12b system to induce staggered DSBs, and localized donor enrichment. In $(\mathrm{a}-\mathrm{c})$, " $\mathrm{P}$ " represents 5 ' -phosphorylation and red " $\mathrm{x}$ " indicates phosphorothioate linkage. (d) Precise genome editing by primer editing. RT: reverse transcriptase. (e) Large sequence insertion using ShCAST. LE: transposon left end; RE: transposon right end.

Lu et al. further applied this approach to improve HDR efficiency. When tandemly repeated sequences are present near DSBs, higher HDR efficiencies have been observed. This is likely due to the repeat sequences being used as a repair template, based on the synthesis-dependent strand annealing (SDSA) mechanism [13]. Therefore, Lu et al. developed a tandem repeat-HDR strategy (TR-HDR) to achieve targeted sequence replacement. A repeat sequence with desired edits is inserted to serve as a template, using the firstly established insertion strategy. At the same time, a target site for the same guide RNA (gRNA) is formed between the two repeats, thus inducing the DSB followed by HDR. This method was successfully used to introduce base substitutions and in-locus tags. The precise editing efficiencies ranged from 3.4 to $11.4 \%$. TR-HDR provided a novel avenue to obtain robust sequence replacement and insertion through HDR.

Collectively, this study made a breakthrough for targeted sequence insertion and replacement in plants that are amenable to biolistic delivery. High editing efficiencies were achieved through NHEJ (25\% on average) and TR-HDR (6.1\% on average), without actively selecting edited sequences, allow- ing complex, precise editing to be efficiently achieved at any desired genomic locus. This simple and reliable strategy was demonstrated to introduce gene regulatory elements, protein tags, and multiple base changes. It could be further applied for gene insertion and replacement, multiplexed single nucleotide polymorphism (SNP) introduction, promoter engineering, etc.

It is notable that this technology can be further improved by overcoming some limitations discussed in this study. The first concern is the random insertion of the repair template and CRISPR reagents into the plant genome, which is also the common problem caused by biolistic delivery. Although this issue can be resolved by generating large $T_{0}$ populations followed by selection, it would be time- and labor-consuming in practice. Using chemically modified ssDNA as the donor is another option to minimize off-target insertions (Figure 1(c)). Although unmodified ssDNA performed poorly in this study, editing efficiency could be significantly increased when using chemically modified templates. In addition, ribonucleoprotein (RNP) delivery of CRISPR reagents has been successfully used 
for plant genome editing to achieve transgene-free genome editing and limit off-target effects [14, 15] (Figure 1(c)). However, RNP-mediated sequence insertion and replacement is still a substantial challenge in plants. The second concern is the chimerism in $T_{0}$ plants, likely because edits happened at late stages of the transgenic plant regeneration. One possible solution is to further improve the editing efficiency. Localized donor enrichment through proteinprotein or protein-DNA interactions can be considered [5, 16-19] (Figure 1(c)). Moreover, Cas12-induced DSBs with staggered ends could potentially facilitate donor insertion (Figure 1(c)).

Recently, CRISPR technologies for sequence insertion and replacement have been advanced rapidly. Prime editing utilizes a reverse transcriptase fused to a Cas9-H840 nickase and a prime editing guide RNA (pegRNA) that encodes the target sequence and the desired edit, to achieve substitutions, deletions, and up to $44 \mathrm{bp}$ insertions [20] (Figure 1(d)). Since prime editing enables flexible genome edits without introducing DSBs and donor templates, it has been applied in plants albeit with low efficiencies in many cases [21-26]. To achieve large sequence insertion, a CRISPR-associated transposase from cyanobacteria Scytonema hofmanni (ShCAST) has been characterized [27] (Figure 1(e)). ShCAST, consisting of Cas12k and three Tn7-like transposase subunits, is able to integrate DNA into specific Escherichia coli genomic sites 60 to $66 \mathrm{bp}$ downstream of the protospacer adjacent motif (PAM) with up to $80 \%$ efficiency [27]. Although this type of DNA insertion is not seamless, it can still be used to insert sequences to introns, around regulatory elements, and in safe harbor loci. However, this system has not been demonstrated in any other organisms. These technologies, along with the one developed by Lu et al., will expand the scope and capabilities of precise and complex genome editing in plants, leading to more biotechnological and agricultural applications.

\section{Disclosure}

The content of this publication is solely the responsibility of the authors and does not necessarily represent the official views of these funding agencies.

\section{Conflicts of Interest}

The authors declare that there is no conflict of interest regarding the publication of this article.

\section{Authors' Contributions}

YZ and YQ participated in the inception, writing, and revising of the manuscript.

\section{Acknowledgments}

This work was supported by the National Science Foundation Plant Genome Research Program grants (award nos. IOS-1758745 and IOS-2029889), Biotechnology Risk Assessment Grant Program competitive grant (award no. 201833522-28789) from the US Department of Agriculture, Syn- genta, and Foundation for Food and Agriculture Research grant (award no. 593603).

\section{References}

[1] Y. Sun, X. Zhang, C. Wu et al., "Engineering herbicideresistant rice plants through CRISPR/Cas9-mediated homologous recombination of acetolactate synthase," Molecular Plant, vol. 9, no. 4, pp. 628-631, 2016.

[2] S. Li, J. Li, J. Zhang et al., "Synthesis-dependent repair of Cpf1induced double strand DNA breaks enables targeted gene replacement in rice," Journal of Experimental Botany, vol. 69, no. 20, pp. 4715-4721, 2018.

[3] S. Li, Y. Zhang, L. Xia, and Y. Qi, "CRISPR-Cas12a enables efficient biallelic gene targeting in rice," Plant Biotechnology Journal, vol. 18, no. 6, pp. 1351-1353, 2020.

[4] Y. Qi, Y. Zhang, F. Zhang et al., "Increasing frequencies of sitespecific mutagenesis and gene targeting in Arabidopsis by manipulating DNA repair pathways," Genome Research, vol. 23, no. 3, pp. 547-554, 2013.

[5] Z. Ali, A. Shami, K. Sedeek et al., "Fusion of the Cas9 endonuclease and the VirD2 relaxase facilitates homology- directed repair for precise genome engineering in rice," Communications Biology, vol. 3, no. 1, p. 44, 2020.

[6] M. Wang, Y. Lu, J. R. Botella, Y. Mao, K. Hua, and J. Zhu, "Gene targeting by homology-directed repair in rice using a geminivirus-based CRISPR/Cas9 system," Molecular Plant, vol. 10, no. 7, pp. 1007-1010, 2017.

[7] T. Dahan-Meir, S. Filler-Hayut, C. Melamed-Bessudo et al., "Efficientin plantagene targeting in tomato using geminiviral replicons and the CRISPR/Cas9 system," The Plant Journal, vol. 95, no. 1, pp. 5-16, 2018.

[8] Q. Shan, N. J. Baltes, P. Atkins et al., “ZFN, TALEN and CRISPR-Cas9 mediated homology directed gene insertion in _Arabidopsis_: A disconnect between somatic and germinal cells," Journal of Genetics and Genomics, vol. 45, no. 12, pp. 681-684, 2018.

[9] S. Li, J. Li, Y. He et al., "Precise gene replacement in rice by RNA transcript-templated homologous recombination," Nature Biotechnology, vol. 37, no. 4, pp. 445-450, 2019.

[10] J. Li, X. Meng, Y. Zong et al., "Gene replacements and insertions in rice by intron targeting using CRISPR- Cas9," Nature Plants, vol. 2, no. 10, p. 16139, 2016.

[11] Y. Lu, Y. Tian, R. Shen et al., "Targeted, efficient sequence insertion and replacement in rice," Nature Biotechnology, pp. $1-6,2020$.

[12] S. Q. Tsai, Z. Zheng, N. T. Nguyen et al., "GUIDE-seq enables genome-wide profiling of off-target cleavage by CRISPR-Cas nucleases," Nature Biotechnology, vol. 33, no. 2, pp. 187-197, 2015.

[13] J. Steinert, S. Schiml, and H. Puchta, "Homology-based double-strand break-induced genome engineering in plants," Plant Cell Reports, vol. 35, no. 7, pp. 1429-1438, 2016.

[14] Z. Liang, K. Chen, T. Li et al., "Efficient DNA-free genome editing of bread wheat using CRISPR/Cas9 ribonucleoprotein complexes," Nature Communications, vol. 8, no. 1, p. 14261, 2017.

[15] S. Svitashev, C. Schwartz, B. Lenderts, J. K. Young, and A. M. Cigan, "Genome editing in maize directed by CRISPRCas9 ribonucleoprotein complexes," Nature Communications, vol. 7, no. 1, p. 13274, 2016. 
[16] K. S. Ghanta, G. A. Dokshin, A. Mir et al., "5’ Modifications improve potency and efficacy of DNA donors for precision genome editing," in bioRxiv, no. article 354480, 2018.

[17] J. Carlson-Stevermer, A. A. Abdeen, L. Kohlenberg et al., "Assembly of CRISPR ribonucleoproteins with biotinylated oligonucleotides via an RNA aptamer for precise gene editing," Nature Communications, vol. 8, no. 1, p. 1711, 2017.

[18] M. Ma, F. Zhuang, X. Hu et al., "Efficient generation of mice carrying homozygous double-floxp alleles using the Cas9-Avidin/biotin-donor DNA system," Cell Research, vol. 27, no. 4, pp. 578-581, 2017.

[19] E. J. Aird, K. N. Lovendahl, A. S. Martin, R. S. Harris, and W. R. Gordon, "Increasing Cas9-mediated homologydirected repair efficiency through covalent tethering of DNA repair template," Communications Biology, vol. 1, no. 1, p. 54, 2018.

[20] A. V. Anzalone, P. B. Randolph, J. R. Davis et al., "Search-andreplace genome editing without double-strand breaks or donor DNA," Nature, vol. 576, no. 7785, pp. 149-157, 2019.

[21] R. Xu, J. Li, X. Liu, T. Shan, R. Qin, and P. Wei, "Development of plant prime-editing systems for precise genome editing," Plant Communications, vol. 1, no. 3, p. 100043, 2020.

[22] H. Butt, G. S. Rao, K. Sedeek, R. Aman, R. Kamel, and M. Mahfouz, "Engineering herbicide resistance via prime editing in rice," Plant Biotechnology Journal, 2020.

[23] X. Tang, S. Sretenovic, Q. Ren et al., "Plant prime editors enable precise gene editing in rice cells," Molecular Plant, vol. 13, no. 5, pp. 667-670, 2020.

[24] Q. Lin, Y. Zong, C. Xue et al., "Prime genome editing in rice and wheat," Nature Biotechnology, vol. 38, no. 5, pp. 582585,2020 .

[25] H. Li, J. Li, J. Chen, L. Yan, and L. Xia, "Precise modifications of both exogenous and endogenous genes in rice by prime editing," Molecular Plant, vol. 13, no. 5, pp. 671-674, 2020.

[26] J. Li, H. Li, J. Chen, L. Yan, and L. Xia, “Toward precision genome editing in crop plants," Molecular Plant, vol. 13, no. 6 , pp. 811-813, 2020.

[27] J. Strecker, A. Ladha, Z. Gardner et al., "RNA-guided DNA insertion with CRISPR-associated transposases," Science, vol. 365 , no. 6448, pp. 48-53, 2019. 